Journal of Advanced Computer Science and Technology, 1 (4) (2012) 240-249

(C) Science Publishing Corporation

www.sciencepubco.com/index.php/JACST

\title{
Hybrid Synchronization of Lorenz-Stenflo System with Delay
}

\author{
Mohammad Ali Khan \\ Department of Mathematics, Garhbeta Ramsundar Vidyabhaban \\ Paschim Medinipur, West Bengal, India. \\ Email:mdmaths@gmail.com
}

\begin{abstract}
This paper presents the hybrid-synchronization between two hyperchaotic Lorenz-Stenflo system with delay. Here we design active controllers so that two pairs of states are synchronized and other two pair of states are anti-synchronized. Numerical results verify that both synchronization and anti-synchronization can co-exist in the hyperchaotic Lorenz stenflo system.
\end{abstract}

Keywords: Hybrid synchronization, Lorenz-Stenflo system, Active control.

\section{Introduction}

The first observation of synchronization was reported by Dutch physicists Christian Huygens in 1665 [1]. In that case, the phenomenon was evidenced by the equal periods of mutually coupled clocks. Subsequently, the synchronization of periodic signals was being detected in many dynamical processes, becoming common in other scientific areas and the subject of various applications in Engineering. Chaos control and synchronization of chaotic systems have been interesting research fields since the pioneering work of Ott, Grebogi and Yorke [2] and the seminal work of Pecora and Carroll [3], which are simultaneously reported in 1990. A variety of approaches has been proposed for the synchronization of chaotic systems which include complete synchronization [3], phase synchronization [4], generalized synchronization [5], anti-synchronization [6], projective synchronization [7], lag synchronization [8-9]. 
Complete synchronization is characterized by the equality of state variables while evolving in time. The anti-synchronization is a phenomenon that the state variable of synchronized systems have the same absolute values but opposite signs, i.e. anti-synchronization is characterized by the dis-appearance of the sum of relevant variables. Projective synchronization is characterized by the fact that the drive and response system could be synchronized upto a scaling factor. Generalized synchronization was discovered in two unidirectionally coupled chaotic systems in 1996, which is defined as the presence of some functional relation between the states of two chaotic systems. At the same time, another regime of chaos synchronization, namely lag synchronization (LS) implies that the state variables of the two coupled systems becomes synchronize but with a time lag with respect to each other. At the begininig, lag synchronization was mostly studied experimentally and numerically. Shahverdiev et.al. [8] have presented the first analytical treatment of lag-synchronization in unidirectionally coupled modified Ikeda models by using Krasovskii-Lyapunov functional approach. In 2009, Tarai et.al. have studied synchronization of bidirectionally coupled chaotic systems [9-10] and also very recently Khan et.al.[11-12] have observed generalized synchronization of bidirectionally coupled chaotic systems via linear transformation. Recently Li [13] and Sudheer et.al. [14] studied hybrid-synchronization behavior in chaotic systems. In hybrid-synchronization one part of the system is synchronized and other part is anti-synchronized.

This paper will focus generalized hybrid-synchronization of hyperchaotic LorenzStenflo system with delay using a nonlinear active control method which is simple, efficient and easy to implement in practical applications. We design active controller so that two pairs of the master and slave systems are synchronized. Finally numerical simulation results are presented to show the efficiency of our proposed method.

\section{System Description}

Here we consider the following four-coupled nonlinear autonomous first order differential equations

$$
\begin{aligned}
\dot{x} & =\alpha(y-x)+\gamma w \\
\dot{y} & =x(r-z)-y \\
\dot{z} & =x y-b z \\
\dot{w} & =-x-\alpha w
\end{aligned}
$$

which are formulated by Stenflo[15] from a low-frequency short wavelength gravity equation. $r(>0), \alpha(>0), \gamma(>0)$ and $\beta(>0)$ are respectively the Rayleigh number, Prandtl number, rotation number and geometric parameter. 
With the following parameters $\alpha=1.0, \beta=0.7, \gamma=1.5$ and $r=26.0$, the Lorenz-Stenflo system exhibits the chaotic motion(Fig.1(a) and (b)).
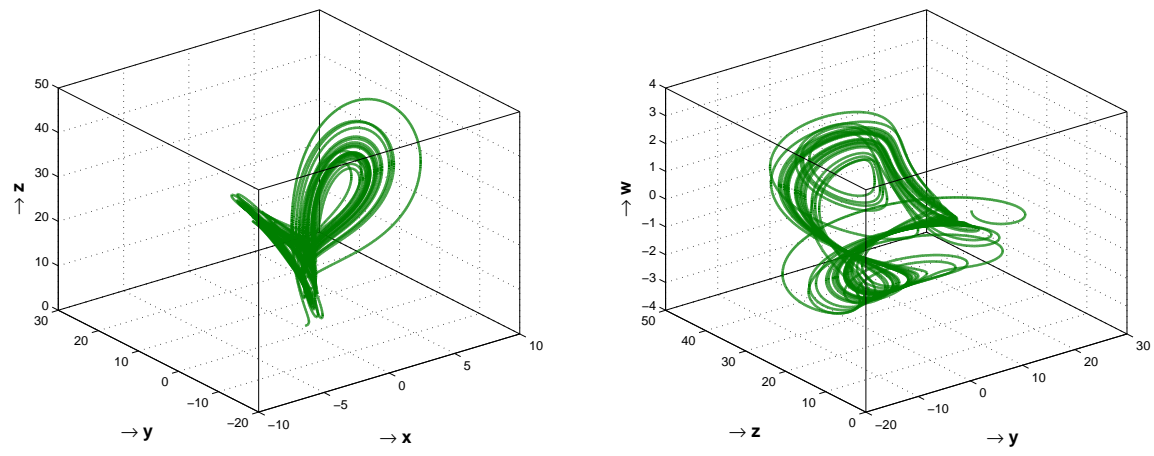

Fig.1(a) and (b): The chaotic motion of Lorenz-Stenflo system at $\alpha=$ $1.0, \beta=0.7, \gamma=1.5$ and $r=26.0$.

\section{Hybrid Synchronization of Lorenz-Stenflo Chaotic System with Delay}

In order to observe the synchronization behavior in the Lorenz-Stenflo system, we have two Lorenz-stenflo systems where the master system with four state variables denoted by the subscript 1 and slave system having identical equations but with delay denoted by the subscript 2 . The master and slave systems are defined as follows:

Master system:

$$
\begin{aligned}
\dot{x}_{1}(t) & =\alpha\left(y_{1}(t)-x_{1}(t)\right)+\gamma w_{1}(t) \\
\dot{y}_{1}(t) & =x_{1}(t)\left(r-z_{1}(t)\right)-y_{1}(t) \\
\dot{z}_{1}(t) & =x_{1}(t) y_{1}(t)-\beta z_{1}(t) \\
\dot{w}_{1}(t) & =-x_{1}(t)-\alpha w_{1}(t)
\end{aligned}
$$

Slave system:

$$
\begin{aligned}
\dot{x_{2}}(t-\tau) & =\alpha\left(y_{2}(t-\tau)-x_{2}(t-\tau)+\gamma w_{2}(t-\tau)+u_{1}\right. \\
\dot{y_{2}}(t-\tau) & =x_{2}(t-\tau)\left(r-z_{2}(t-\tau)\right)-y_{2}(t-\tau)+u_{2} \\
\dot{z}_{2}(t-\tau) & =x_{2}(t-\tau) y_{2}(t-\tau)-\beta z_{2}(t-\tau)+u_{3} \\
\dot{w}_{2}(t-\tau) & =-x_{2}(t-\tau)-\alpha w_{2}(t-\tau)+u_{4}
\end{aligned}
$$




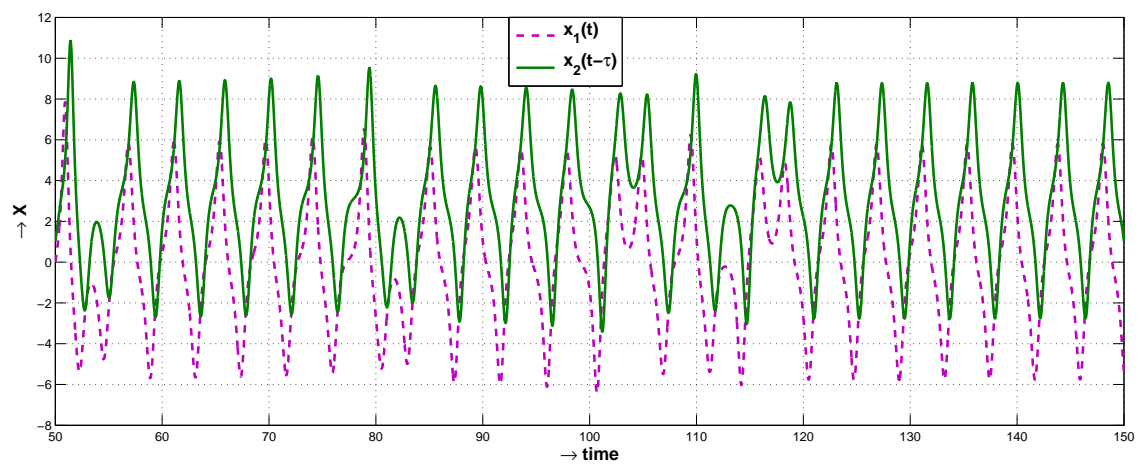

Fig.2(a): Time evolution of $x_{1}$ and $x_{2}$ when active control law deactivated.

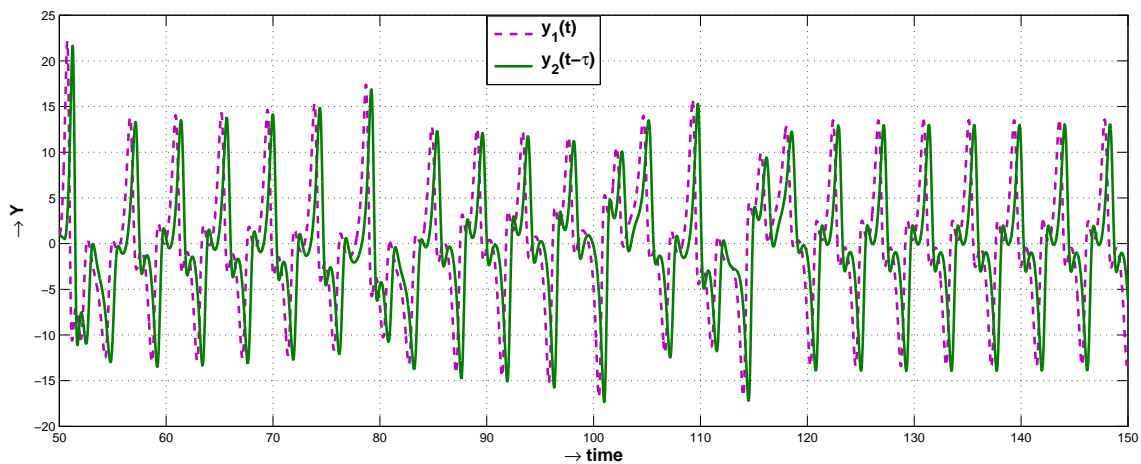

Fig.2(b): Time evolution of $y_{1}$ and $y_{2}$ when active control law deactivated.

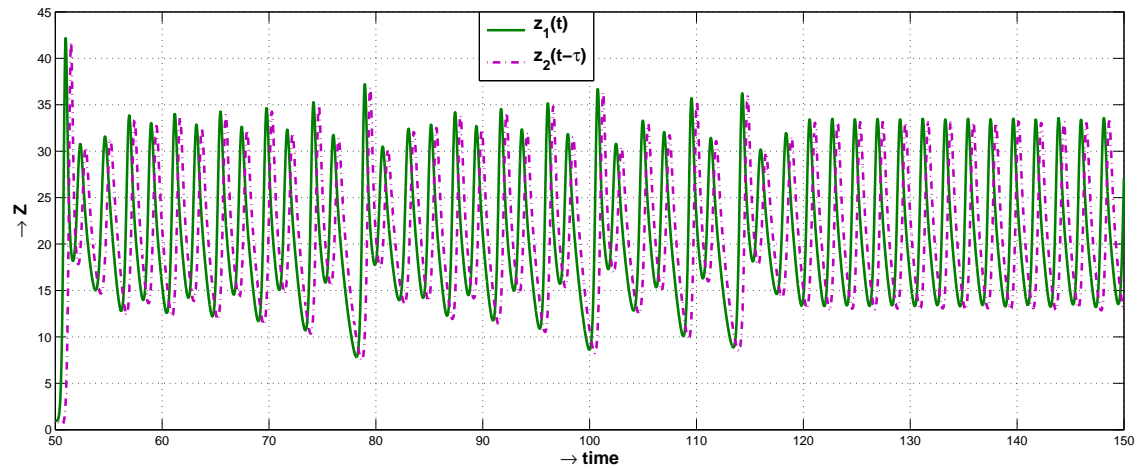

Fig.2(c): Time evolution of $z_{1}$ and $z_{2}$ when active control law deactivated. 


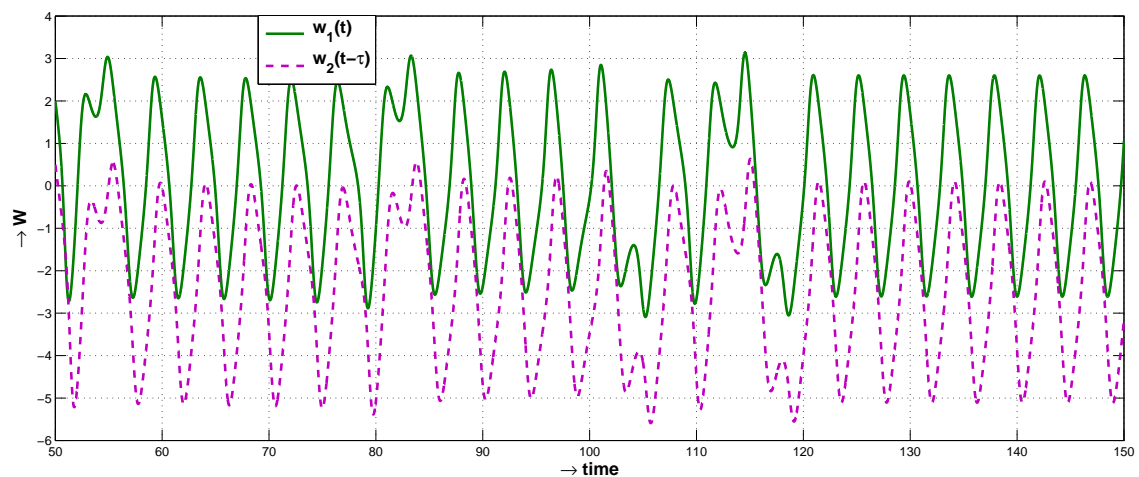

Fig.2(d): Time evolution of $w_{1}$ and $w_{2}$ when active control law deactivated.

We have introduced four control functions $u_{1}, u_{2}, u_{3}$ and $u_{4}$. These control functions are to be determined for the purpose of hybrid synchronization of the two systems with the same parameter and different initial conditions. For the hybrid synchronization, we define the state errors between the slave system that is to be controlled and the controlling master system as

$$
\begin{aligned}
e_{1}=x_{2}(t-\tau)-x_{1}(t) ; e_{2} & =y_{2}(t-\tau)+y_{1} ; e_{3}=z_{2}(t-\tau)-z_{1} \text { and } \\
e_{4} & =w_{2}(t-\tau)+w_{1}
\end{aligned}
$$

Using this notation, we obtain

$$
\begin{aligned}
& \dot{e_{1}}=\alpha\left(e_{2}-e_{1}\right)+\gamma e_{4}-2 \alpha y_{1}(t)-2 \gamma w_{1}(t)+u_{1} \\
& \dot{e_{2}}=-e_{2}+r e_{1}+2 r x_{1}(t)-x_{1}(t) z_{1}(t)-x_{2}(t-\tau) z_{2}(t-\tau)+u_{2} \\
& \dot{e_{3}}=-\beta e_{3}+x_{2}(t-\tau) y_{2}(t-\tau)-x_{1} y_{1}+u_{3} \\
& \dot{e_{4}}=-e_{1}-\alpha e_{4}-2 x_{1}(t)+u_{4}
\end{aligned}
$$

We choose the active control function $u=\left[u_{1}, u_{2}, u_{3}, u_{4}\right]^{T}$ as

$$
\begin{aligned}
& u_{1}=2 \alpha y_{1}(t)+2 \gamma w_{1}(t)+v_{1} \\
& u_{2}=x_{1}(t) z_{1}(t)+x_{2}(t-\tau) z_{2}(t-\tau)-2 r x_{1}(t)+v_{2} \\
& u_{3}=x_{1}(t) y_{1}(t)-x_{2}(t-\tau) y_{2}(t-\tau)+v_{3} \\
& u_{4}=2 x_{1}(t)+v_{4}
\end{aligned}
$$

Then the error system becomes

$$
\begin{aligned}
\dot{e_{1}} & =\alpha\left(e_{2}-e_{1}\right)+\gamma e_{4}+v_{1} \\
\dot{e_{2}} & =-e_{2}+r e_{1}+v_{2} \\
\dot{e_{3}} & =-\beta e_{3}+v_{3} \\
\dot{e_{4}} & =-e_{1}-\alpha e_{4}+v_{4}
\end{aligned}
$$


Thus the system (6) to be controlled in a linear system with the control input $v=\left(v_{1}, v_{2}, v_{3}, v_{4}\right)^{T}$ as functions of the error states $e_{1}, e_{2}, e_{3}, e_{4}$, then (6) is stabilized by the feedback $v$, then the error will converge to zero as time goes to infinity, which implies that the systems (2) and (3) are globally synchronized. We choose $v$ such that

$$
\left[v_{1}, v_{2}, v_{3}, v_{4}\right]^{T}=A\left[e_{1}, e_{2}, e_{3}, e_{4}\right]
$$

Where $A$ is a $4 \times 4$ matrix. For (8) to be asymptotically stable, the feedback system of the element of the matrix $A$ must have all of its eigenvalues with negative real parts. Here we choose the matrix $A$ as

$$
A=\left(\begin{array}{cccc}
0 & -2 \alpha & 0 & 0 \\
r & -1 & 0 & 0 \\
1 & 0 & 2 & 5 \alpha
\end{array}\right)
$$

Hence the error system becomes

$$
\begin{aligned}
& \dot{e_{1}}=\alpha\left(-e_{2}-e_{1}\right)+\gamma e_{4} \\
& \dot{e_{2}}=-e_{2}+r e_{1} \\
& \dot{e_{3}}=-\beta e_{3}-e_{4} \\
& \dot{e_{4}}=2 e_{3}+4 \alpha e_{4}
\end{aligned}
$$

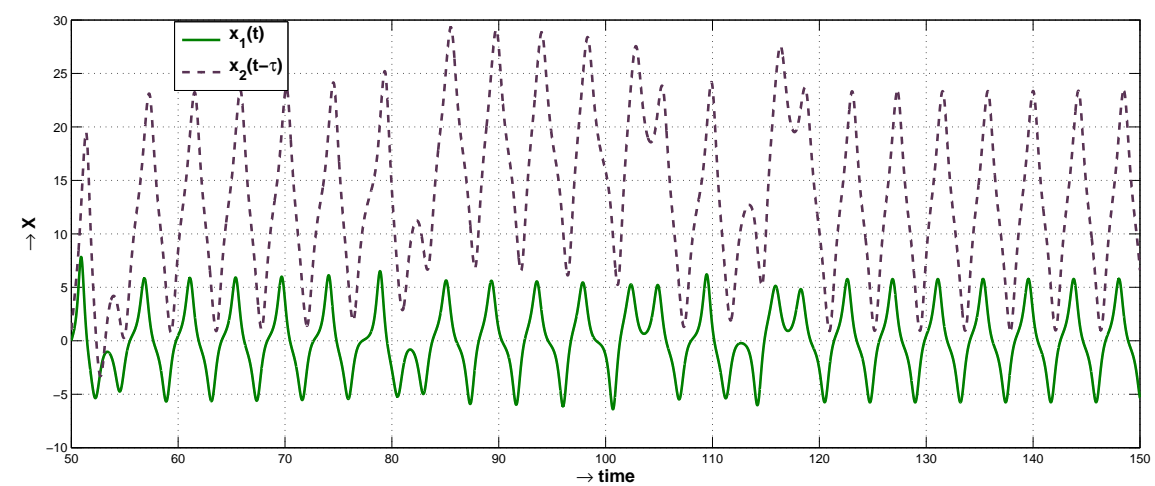

Fig.3(a): Time evolution of $x_{1}$ and $x_{2}$ when active control law activated. 


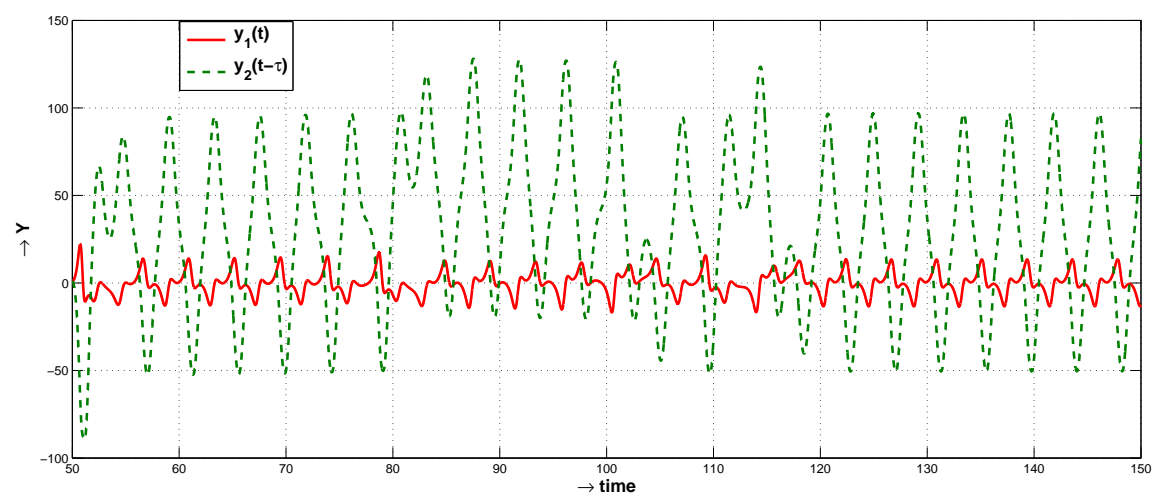

Fig.3(b): Time evolution of $y_{1}$ and $y_{2}$ when active control law activated.

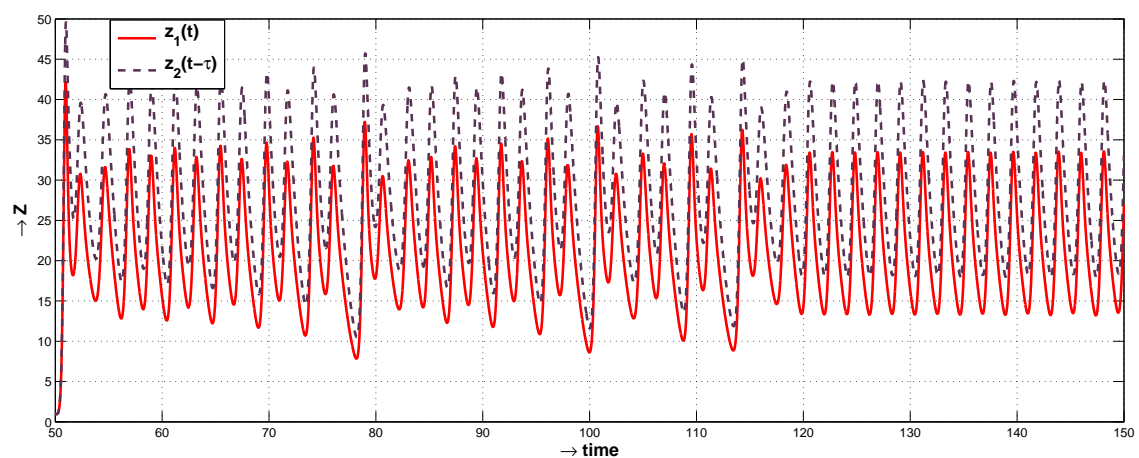

Fig.3(c): Time evolution of $z_{1}$ and $z_{2}$ when active control law activated.

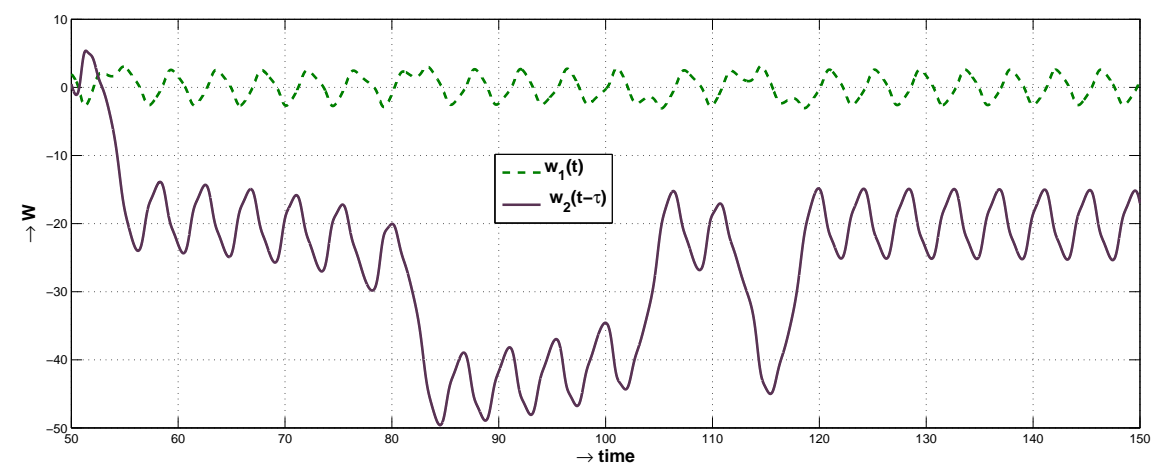

Fig.3(d): Time evolution of $w_{1}$ and $w_{2}$ when active control law activated. 


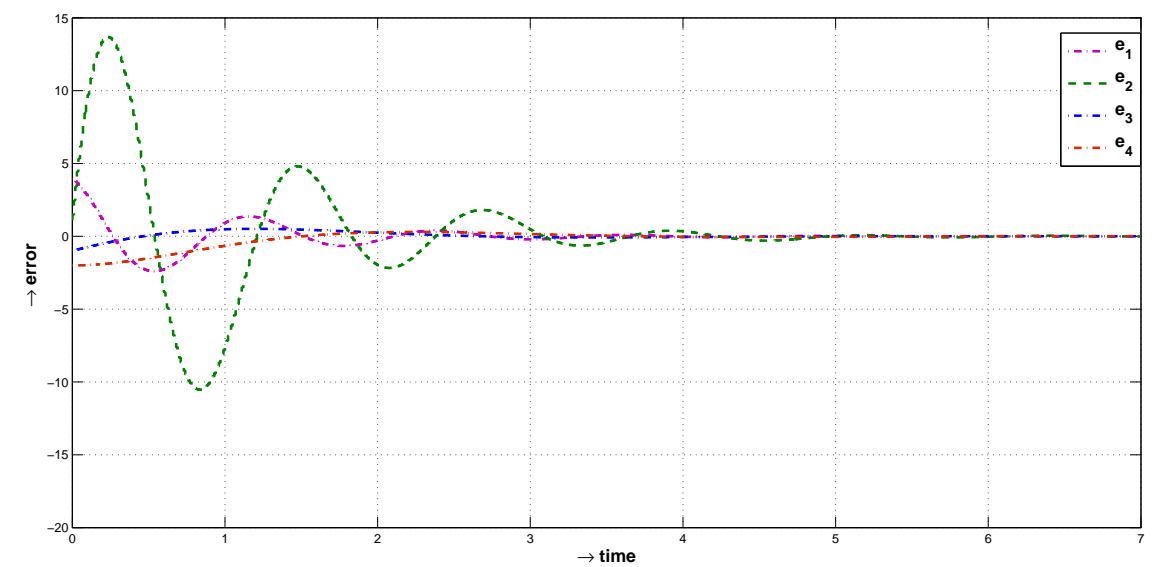

Fig.4: Time evolution of error systems when active control law activated.

\section{$4 \quad$ Simulation Results}

Numerical simulations have been performed for demonstration of the hybrid synchronization method. Fourth-order Runge-Kutta method is used for solving Lorenz-Stenflo system with delay with active control and without active control. Lorenz-Stenflo system is chaotic for $\alpha=1.0, \beta=0.7, \gamma=1.5$ and $r=26.0$ (Figures 1(a) and 1(b)). The initial values for the drive and response systems are $x_{1}(0)=0.0, y_{1}(0)=1.0, z_{1}(0)=1.0, w_{1}(0)=2.0$ and $x_{2}(0)=1.0$, $y_{2}(0)=1.0, z_{2}(0)=1.0, w_{2}(0)=0.5$ with $\tau=0.5$. Figures $2(\mathrm{a})-2(\mathrm{~d})$ shows the time response of states for the drive system and response system when active control is deactivated and Figures 3(a)-3(d)depicts the same signal when active control activated. Figure 4. display the time response of the error system.

\section{Conclusions}

In this work demonstrates the hybrid synchronization between two hyperchaotic Lorenz-Stenflo systems through active control method. Numerical simulations are shown to be consistent with theoretical statements.

\section{References}

[1] C. Huygens, Letter to de sluse, In: Oeuveres completes de Christian Huygens, Societe Hollandaise Des Sciences, Martinus Nijhoff, La Haye (1665). 
[2] E. Ott, C. Grebogi and JA Yorke, Controlling chaos, Phys.Rev.Lett., 64 (1990)1196-1199.

[3] LM. Pecora and TL. Carroll, Synchronization in chaotic systems, Phys.Rev.Lett., 64 (1990)821-824.

[4] B. Schelter, M. Winterhaldar and J. Timmer, Phase synchronization and coherence analysis: sensitivity and specificity, International Journal of Bifurcation and Chaos, 17(2007)3551-3556.

[5] L. Kocarev and U. Parlitz, Generalized synchronization, predictability, an equivalence of unidirectionally coupled dynamical systems, Phys.Rev.Lett., 76 (1996)1816-1819.

[6] M.A. Khan, S.N. Pal and S. Poria, Generalized anti-synchronization of different chaotic systems, Int. J. of Applied Mechanics and Engineering, 17(2012)83-89.

[7] R. Mainieri and J. Rahacek, Projective synchronization in three dimensional chaotic systems, Phys. Rev. Lett., 82 (1999)3042-3045.

[8] EM. Shahverdiev, S. Sivaprakasam and K.A. Shore, Lag synchronization in time delayed systems, Phys.Lett.A, 292(2002)320-324.

[9] A. Tarai, S.Poria and P. Chatterjee, Synchronization of bidirectionally coupled chaotic Chen's system with delay, Chaos Solitons and Fractals., 41 (2009)643-647.

[10] A. Tarai, S.Poria and P. Chatterjee, Synchronization of generalized linearly bidirectionally coupled unified chaotic system, Chaos Solitons and Fractals., 40 (2009)885-892.

[11] M.A. Khan, Generalized synchronization of bidirectionally coupled chaotic systems via linear transformation, Int. J. of Basic and Applied Sciences, 1(2012)270-282.

[12] M.A. Khan and S. Poria, Generalized synchronization of bidirectionally coupled chaotic systems, Int. J. of Applied Mathematical Research,1(2012)303-313.

[13] RH. Li, A special full-state hybrid-synchronization in symmetrical chaotic system, Applied Math.Comput.200(2008)319-321.

[14] K.S. Sudheer and M. Sabir, Hybrid synchronization of hyperchaotic Lu system, PRAMANA-journal of physics, 73(2009)781-786. 
[15] L. Stenflo, Generalized Lorenz equations for Acoustic-Gravity waves in the atmosphere, Physica Scripta, 53(1996)83-84. 